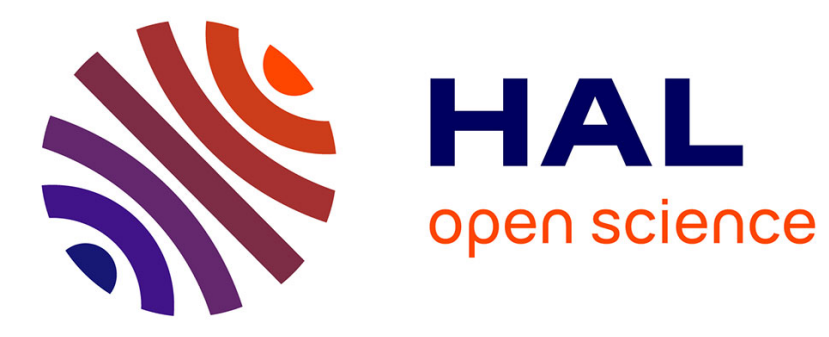

\title{
Strategic influence in social networks
}

Michel Grabisch, Antoine Mandel, Agnieszka Rusinowska, Emily Tanimura

\section{To cite this version:}

Michel Grabisch, Antoine Mandel, Agnieszka Rusinowska, Emily Tanimura. Strategic influence in social networks. 2015. hal-01158168

\section{HAL Id: hal-01158168 \\ https://hal.science/hal-01158168}

Submitted on 29 May 2015

HAL is a multi-disciplinary open access archive for the deposit and dissemination of scientific research documents, whether they are published or not. The documents may come from teaching and research institutions in France or abroad, or from public or private research centers.
L'archive ouverte pluridisciplinaire HAL, est destinée au dépôt et à la diffusion de documents scientifiques de niveau recherche, publiés ou non, émanant des établissements d'enseignement et de recherche français ou étrangers, des laboratoires publics ou privés. 


\section{Documents de Travail du

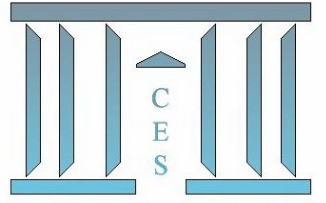

Strategic influence in social networks

Michel GRABISCH, Antoine MANDEL,

Agnieszka RUSINOWSKA, Emily TANIMURA

2015.06

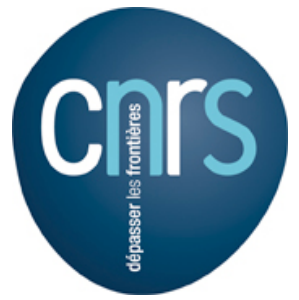

Maison des Sciences Économiques, 106-112 boulevard de L'Hôpital, 75647 Paris Cedex 13 


\title{
Strategic influence in social networks ${ }^{\star}$
}

\author{
Michel Grabisch ${ }^{1}$, Antoine Mandel ${ }^{1 \star \star}$, Agnieszka Rusinowska ${ }^{2}$, and Emily Tanimura ${ }^{3}$ \\ 1 Paris School of Economics, Université Paris I Panthéon-Sorbonne \\ Centre d'Economie de la Sorbonne, 106-112 Bd de l'Hôpital, 75647 Paris Cedex 13, France \\ michel.grabisch@univ-paris1.fr \\ antoine.mandel@univ-paris1.fr \\ 2 Paris School of Economics - CNRS, Université Paris I Panthéon-Sorbonne, Centre d'Economie de la Sorbonne \\ agnieszka.rusinowska@univ-paris1.fr \\ 3 Université Paris I Panthéon-Sorbonne, Centre d'Economie de la Sorbonne \\ Emily.Tanimura@univ-paris1.fr
}

\begin{abstract}
We consider a model of influence with a set of non-strategic agents and two strategic agents. The non-strategic agents have initial opinions and are linked through a simply connected network. They update their opinions as in the DeGroot model. The two strategic agents have fixed opinions, 1 and 0 respectively, and are characterized by the magnitude of the impact they can exert on non-strategic agents. Each strategic agent forms a link with one non-strategic agent in order to alter the average opinion that eventually emerges in the network. This procedure defines a zero-sum game whose players are the two strategic agents and whose strategy set is the set of non-strategic agents. We focus on the existence and the characterization of equilibria in pure strategy in this setting. Simple examples show that the existence of a pure strategy equilibrium does depend on the structure of the network. The characterization of equilibrium we obtain emphasizes on the one hand the influenceability of target agents and on the other hand their centrality whose natural measure in our context defines a new concept, related to betweenness centrality, that we call intermediacy. We also show that in the case where the two strategic agents have the same impact, symmetric equilibria emerge as natural solutions whereas in the case where the impacts are uneven, the strategic players generally have differentiated equilibrium targets, the high-impact agent focusing on centrality and the low-impact agent on influenceability.
\end{abstract}

JEL Classification: C71, D85

Keywords: influence network, beliefs, DeGroot model, strategic player, convergence, consensus, equilibrium

\section{Introduction}

Opinion formation and influence are faithful companions of most dynamic interactions between individuals. Since any interaction within a group of individuals can be represented by a network structure, it is not surprising that numerous models of influence in social networks have been proposed in the literature. For surveys on belief and opinion dynamics in social networks we refer, e.g., to Jackson (2008) and Acemoglu and Ozdaglar (2011). These formal models can be used to understand how different assumptions about the interactions affect the opinions that emerge and in particular to understand when interacting individuals with different opinions or beliefs will ultimately reach a consensus.

However, one aspect of social learning and influence in networks that has not received much attention is that of individual strategy. Often, the role of the interacting individuals

\footnotetext{
^ This research is supported by the European Project SIMPOL (Financial Systems Simulation and Policy Modeling).

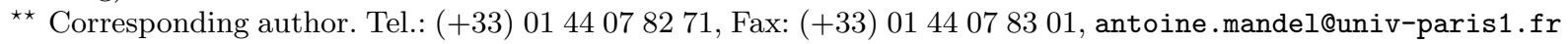


is merely that of a passive vector for a diffusion process which causes them to adopt and pass on informations and opinions they receive. In reality, it seems likely that (at least some) individuals have their own convictions and agendas and will seek to impose these in a social exchange. In the present paper, we present a model which, while remaining close to the seminal framework of DeGroot (1974) addresses this issue.

Considering agents who are both strategic and susceptible to influence leads to obvious difficulties in evaluating payoffs. Indeed, if an agent succeeds in imposing his initial opinion but has since then changed opinions himself, it is not straightforward how he should evaluate the outcome. For this reason, it seems natural to consider a model involving two types of agents: strategic and non strategic ones. Strategic agents with conflicting opinions attempt to optimally target non strategic agents to spread their own opinion. More precisely, we consider a set of non-strategic agents characterized by a vector of opinions (beliefs), where an opinion is a number in $[0,1]$. These agents are permanently linked through a strongly connected network and each agent updates his opinion by taking a weighted average of the opinions of his neighbors, similarly to the model of DeGroot (1974). Additionally, we assume that there are two 'external' agents (we call them strategic agents), with the opposite opinions 1 and 0 , and with some (possibly different) relative weights. These strategic players target some of the non-strategic agents in order to alter the average opinion that eventually emerges in the network. More precisely, each strategic agent chooses exactly one non-strategic agent and forms a link with him to influence his opinion, and consequently, the final vector of opinions, if convergence occurs. We prove that convergence does occur.

By defining for each player a payoff based on his success in influencing the limit opinion, we obtain a zero sum game whose players are the strategic agents and in which the set of strategies is the choice of non-strategic agents. In a certain sense, the problem we consider is similar to that of optimal targeting, in order to maximize the spread of something in a network. However, the optimal targeting problem which has been extensively studied in the literature, and which we discuss in detail in the next section, is an optimization problem. When two players with conflicting interests choose targets, optimization based on network structure is combined with strategic considerations. These two aspects are reflected in our results.

First, we provide simple examples to show that the existence of a pure strategy equilibrium does depend on the structure of the network. For example, there is no equilibrium in pure strategy in the circle because if the target of an agent is fixed, his opponent can optimally undercut him by targeting the agent "next in line". On the contrary, in symmetric networks, every choice of a target is an equilibrium.

Second, we derive a general expression of the payoff of the strategic agent and a characterization of equilibria in pure strategies. This characterization stresses two features of target agents: their influenceability and their centrality (i.e the influence they exert). Influenceability is measured via the number of outgoing links: the less opinions within the network a non-strategic agent take into account, the more she can be influenced by the strategic agents. As for centrality or influence, a new measure that we call intermediacy naturally emerges in our framework: it counts the number of walks that go through the agent. When the strategic agents exert an equal impact, we show that symmetric equilibria naturally emerge and that the equilibrium target strikes the balance between influenceability and influence that is appropriate given the size of the network and the 
weight of strategic agents. Influenceability gains importance vis-à-vis influence as the size of the network grows or the impact of the strategic agent decreases. In the case where the impact of the two strategic agents are uneven, they generally have different targets at equilibrium. Indeed, the influence of the low-impact agent would be washed away if he targeted the same agent as his opponent. As a matter of fact, at equilibrium, the target choice of the high-impact agent is driven by centrality and this of the low-impact agent by influenceability.

The remaining part of the paper is structured as follows. Section 2 presents an overview of related literature on opinion formation. In Section 3 we recall the DeGroot model and some results on nonnegative matrices that are then applied to the DeGroot model and to our framework. The model of strategic formation of influence networks with two strategic agents is formally introduced and its convergence is discussed in Section 4 . The properties of the Nash equilibrium of the model are studied in Section 5, which also discusses the case of a single strategic agent. Section 6 concludes the paper with some final remarks.

\section{Related literature on opinion and belief dynamics}

As mentioned in Acemoglu and Ozdaglar (2011), one can classify models of opinion formation according to the way information is conveyed (does an individual update his opinion by observing other individuals or rather by communicating with them) and the way it is processed (do individuals use Bayes rule or not in the updating process). In the Bayesian literature, we find models of observation-based learning (e.g., herd behavior of Banerjee (1992), informational cascades of Bikhchandani et al. (1992), see also Ellison and Fudenberg (1993); Banerjee and Fudenberg (2004); Bala and Goyal (1998); Acemoglu et al. (2011)) as well as models of communication-based learning (e.g., Hagenbach and Koessler (2010); Galeotti et al. (2013)). In the non-Bayesian literature, to which the present paper belongs, a standard approach to opinion formation is imitation where an agent uses a combination of beliefs of some others that he has observed (e.g., DeGroot (1974); DeMarzo et al. (2003); Golub and Jackson (2010)). Other non-Bayesian approaches have their origin in models of interacting particle systems from statistical mechanics. In these models, opinions can be represented by finitely many discrete values (e.g. in the Ising model of Glauber (1963) and the voter model of Clifford and Sudbury (1973)) or continuous values (e.g., Deffuant et al. (2000); DeGroot (1974); Krause (2000); Hegselmann and Krause (2002)).

We are particularly interested in the seminal model of belief and consensus formation by DeGroot (1974), where each agent updates his opinion (which is a number in $[0,1]$ ) by a weighted average of the opinions of the agents. The interaction among players is captured by the social influence matrix. Convergence of opinions is obtained under mild conditions on the interaction structure (strong connectedness and aperiodicity). Different extensions of the DeGroot model have been proposed in the literature, in particular, models where the updating can vary with time and circumstances, e.g., Krause (2000) (only weighting those with similar beliefs), DeMarzo et al. (2003) (time-varying weight on own beliefs) and models presented in Friedkin and Johnsen $(1990,1997)$. With regards to the aggregation of opinions, Grabisch and Rusinowska (2013) investigate a dynamic stochastic model of influence where arbitrary aggregation functions (not only weighted averages) can be used to update agents' opinions, and Förster et al. (2013) investigate influence processes 
modeled by ordered weighted averaging operators which allow us to study anonymous influence (aggregation depends only on how many agents hold an opinion instead of which agents do so). Other studies on consensus and convergence of opinion dynamics include that of Hegselmann and Krause (2002); Lorenz (2005, 2006); Golub and Jackson (2010). More closely related to this paper, strategic extensions of the DeGroot model are considered in Büchel et al. (Forthcoming) where agents interact strategically in the discussion and may misrepresent their opinion in a conforming or counter-conforming way, and in Büchel et al. (2014) agents strategically interact via expressed opinions, i.e., the transmission of cultural traits from one generation to the next.

Our paper adds two particular issues to this strand of literature. First, the existence of different types of agents, with the particular case that some agents influence others without themselves being influenced. Second, the targeting of specific (central) individuals in social networks. In this respect, there exist several works in the literature in economics and computer sciences to which our paper is related.

Acemoglu et al. (2010) consider a variation on the DeGroot model by assuming that there are two types of agents: regular and forceful. While regular agents exchange information with their neighbors when they meet, forceful agents influence others disproportionately. The authors use an asynchronous continuous-time model to represent meetings between agents. Also Acemoglu et al. (2013) study an opinion dynamics model with two types of agents: regular and stubborn. Regular agents update their beliefs according to information that they receive from the neighbors and stubborn agents never update their opinions. When the society contains stubborn agents with different opinions, a consensus is never reached. Our approach is related to these works as we also consider two types of agents but our key concern is the strategic nature of some of the agents, which is not covered in the work of Acemoglu and co-authors.

The problem of targeting the right individuals is a major concern in marketing or in lobbying and has been extensively studied theoretically in computer science. Which set of individuals should we target if we aim to have a large cascade of adoptions of a new product or innovation? Domingos and Richardson (2001) and Richardson and Domingos (2002) pose a fundamental algorithmic problem for such social network processes. Also Kempe et al. (2003) study this problem and consider the task of choosing influential sets of individuals as a problem in discrete optimization. The authors focus on models based on the use of node-specific thresholds (Granovetter (1978); Schelling (1978)), and also consider dynamic cascade models for diffusion processes based on works in interacting particle systems (Durrett (1988); Liggett (1985)). They show that approximation algorithms for maximizing the spread of influence in these models can be developed in a general framework based on submodular functions. Furthermore, the authors provide computational experiments on large collaboration networks and show that the proposed algorithm achieves significant performance gains over degree centrality and closeness centrality. Similarly, Kempe et al. (2005) study the problem of target selection in order to maximize the expected spread of an innovation or of some behavior within a social network in the "word-of-mouth" referral framework. The authors develop a more general model of influence propagation (decreasing cascade model) where starting with a set of initially "active" nodes, a behavior spreads in a cascading fashion according to a probabilistic rule. Which set of nodes should we target for initial activation to maximize the expected size of the cascade? 
In the economics literature, we also find studies of optimal targeting in the context of local interaction. Ballester et al. (2006) analyze a noncooperative game interpreted as a network game with local payoff complementarities, where players decide how much effort to exert. In such a game, the Nash equilibrium action of a player is proportional to his Bonacich centrality (Bonacich (1987)). The authors study a policy for targeting the key player, that is, a player whose removal from the network leads to the optimal change in aggregate activity. They provide a characterization of the key player identified with an "intercentrality measure" that takes into account both a player's centrality and his contribution to the centrality of the others.

Galeotti and Goyal (2009) model networks in terms of degree distributions and study influence strategies in the presence of local interaction. In their model there are two groups of players, and every member of the first group chooses a strategy to influence members of the second group. The actions taken by the members of the first group lead to some information that is shared locally by the members of the second group. A new distribution of information is obtained and the members of the second group make decisions based on this distribution, which generates payoffs for members in the first group. The authors examine how social networks affect the optimal social influence decisions of players in the first group.

The problem of identifying central agents in a social network is also studied in Banerjee et al. (2014). They analyze a "gossip" process, where players generate some information about particular people which is then stochastically passed from neighbor to neighbor. The authors use a specific measure of a player's ability to send information called "diffusion centrality" (Banerjee et al. (2013)) which measures how widely the information spreads from a given player in a given number of time periods and for a given random per period transmission probability. The diffusion centrality nests degree centrality (if there is one time period of communication), eigenvector centrality and Katz-Bonacich centrality (if there are unlimited periods of communication).

\section{Nonnegative matrices and the DeGroot model}

\subsection{General results on nonnegative matrices}

We recall general results on nonnegative matrices given in Seneta (2006). By convention, transposition is denoted by ', and hence row vectors are marked with ', like $v^{\prime}$.

We set $N=\{1, \ldots, n\}$ and consider nonnegative $n \times n$ matrices. The $k$ th power of such a matrix $T=\left[t_{i j}\right]$ is denoted by $T^{k}=\left[t_{i j}^{(k)}\right]$. To any nonnegative matrix $T$ we associate a directed graph $\Gamma$ with set of nodes $N$, and an arc $(i, j)$ exists iff $t_{i j}>0$. A walk of length $k$ from node $i$ to node $j$ is a sequence of nodes $i=i_{0}, i_{1}, \ldots, i_{k}=j$ such that $\left(i_{\ell-1}, i_{\ell}\right)$ is an arc in $\Gamma$ for $\ell=1, \ldots, k$. Note that the existence of such a walk is equivalent to $t_{i j}^{(k)}>0$. We define cycles as walks from a node to itself. We write $i \rightarrow j$ if there is a walk from $i$ to $j$. We also use the notation $i \not \rightarrow j$ and $i \leftrightarrow j$.

A class or strongly connected component of $\Gamma$ is a set of nodes $C$ such that either $C$ is a singleton or $i \leftrightarrow j$ for every distinct $i, j \in C$, and any $C^{\prime} \supset C$ does not fulfill the latter property. A class can be essential if no arc is going out of it, otherwise it is inessential. 
The canonical form of a matrix $T$ with $q$ essential classes and $w$ inessential classes is

$$
T=\left[\begin{array}{cccc|c}
T_{1} & 0 & \cdots & 0 & \\
0 & T_{2} & \ddots & \vdots & \\
\vdots & \ddots & \ddots & 0 \\
0 & \cdots & 0 & T_{q} & \\
\hline \multicolumn{4}{c}{R} & Q
\end{array}\right]
$$

with

$$
Q=\left[\begin{array}{cccc}
Q_{1} & 0 & \cdots & 0 \\
& Q_{2} & & \vdots \\
\vdots & & \ddots & \\
S & \cdots & & Q_{w}
\end{array}\right]
$$

where elements in $N$ have been ordered so that essential classes come first (in any order), then inessential classes, so that if for $i, j$ in two distinct inessential classes, $i$ is ranked before $j$, we have $i \not \rightarrow j$. We have

$$
T^{k}=\left[\begin{array}{cccc|c}
T_{1}^{k} & 0 & \cdots & 0 & \\
0 & T_{2}^{k} & \ddots & \vdots & \\
\vdots & \ddots & \ddots & & 0 \\
0 & \cdots & 0 & T_{q}^{k} & \\
\hline \multicolumn{4}{c}{R_{k}} & Q_{k}
\end{array}\right]
$$

with

$$
Q_{k}=\left[\begin{array}{cccc}
Q_{1}^{k} & 0 & \cdots & 0 \\
& Q_{2}^{k} & & \vdots \\
\vdots & & \ddots & \\
S_{k} & \cdots & & \\
& & & \\
w
\end{array}\right]
$$

where $S_{k}, R_{k}$ are in general difficult to compute.

The period $d(i)$ of an element $i \in N$ such that $i \rightarrow i$ is the greatest common divisor of those $k$ for which $t_{i i}^{(k)}>0$ (i.e., $k=$ length of a cycle on $i$ ). If $i \leftrightarrow j$ then $d(i)=d(j)$, so that one can speak of the period of a class. A class is aperiodic if $d(i)=1$ for some $i$ in the class.

A matrix $T$ is primitive if $T^{k}>\mathbf{0}$ for some integer $k$. It is irreducible if for every $i, j \in N$ there exists an integer $m(i, j)$ such that $t_{i j}^{(m(i, j))}>0$. An irreducible matrix corresponds to a single class. $T$ is primitive iff $T$ is irreducible and aperiodic.

In the rest of the section we recall some important facts.

Fact 1 The Perron-Frobenius theorem holds for irreducible matrices $T$ : there exists a positive real eigenvalue $r$, which can be associated with unique (up to a multiplicative constant) positive left and right eigenvectors, and such that $r \geq|\lambda|$ for every other eigenvalue $\lambda$. The eigenvalue $r$ is a simple root of the characteristic equation of $T$. Moreover, if the period of the class is $d$, there are exactly $d$ eigenvalues $\lambda$ such that $|\lambda|=r$. These eigenvalues are $\lambda_{k}=r \cdot e^{\frac{2 \pi i k}{d}}, k=1, \ldots, d$. Finally, a useful result is that

$$
\min _{i} \sum_{j=1}^{n} t_{i j} \leq r \leq \max _{i} \sum_{j=1}^{n} t_{i j}
$$


(idem for columns), with equality on one side implying equality throughout.

Let us then consider a row-stochastic matrix $P$. If $P$ is irreducible, then by (4), 1 is the Perron-Frobenius eigenvalue, and there is a unique left eigenvector $v^{\prime}$ (i.e., $v^{\prime} T=v^{\prime}$ ) such that $v^{\prime} \cdot \mathbf{1}=1$. With notation of $(1)$, we have the following:

Fact 2 If $P$ is primitive, then $\lim _{k \rightarrow \infty} P^{k}=\mathbf{1} \cdot v^{\prime}$.

Fact $3 \lim _{k \rightarrow \infty} Q^{k}=\mathbf{0}$

Fact 4 Interpreting $p_{i j}$ as the probability to go from state $i$ to state $j$, we have

$$
x_{\rho}=[I-Q]^{-1} x_{\rho}^{(1)}
$$

where $x_{\rho} \in[0,1]^{n}, x_{\rho, i}$ is the probability to finish in class $\rho$, starting from the inessential state $i$, and $x_{\rho, i}^{(1)}=\sum_{j \in E_{\rho}} p_{i j}$ is the probability of absorption in class $\rho$ after one step, starting from $i$.

The matrix $[I-Q]$ is the fundamental matrix of absorbing chains. We recall:

Lemma 1 Let $A$ be a finite $n \times n$ matrix such that $\lim _{k \rightarrow \infty} A^{k}=\mathbf{0}$. Then $[I-A]^{-1}$ exists and

$$
[I-A]^{-1}=\sum_{k=0}^{\infty} A^{k}
$$

with $A^{0}=I$.

\subsection{The DeGroot model}

We recall the seminal model of opinion formation and influence introduced in DeGroot (1974). Consider a set $N=\{1, \ldots, n\}$ of agents interacting in a social network. Each agent $i$ has an initial opinion (belief) on a subject denoted by $x_{i}(0) \in[0,1]$ and updates his opinion at discrete time instances. The interactions between the agents are represented by a nonnegative $n \times n$ row-stochastic matrix $W=\left[w_{i j}\right]$, where $w_{i j}$ denotes the weight (or trust) that agent $i$ places on the current opinion of agent $j$ in forming his own opinion in the next period. The opinions are updated according to

$$
x(t)=W x(t-1)=W^{t} x(0)
$$

where $x(t)=\left[x_{1}(t), \ldots, x_{n}(t)\right]^{\prime}$ is the opinion vector at time step $t$. Hence, agents update their opinions by a convex combination of their current opinions and the opinions of their neighbors. We associate to the matrix $W$ a directed graph $\Gamma$ such that there is an arc from $i$ to $j$ iff $w_{i j}>0$.

We can apply the results recalled in Subsection 3.1 to the DeGroot model. The matrix $W$ given in (5) can be put under the form (1) and (2), after identification of the essentials and inessential classes. We write $x=\left[\begin{array}{ll}x_{E} & x_{I}\end{array}\right]^{\prime}=\left[\begin{array}{llllll}x_{E_{1}} & \cdots & x_{E_{q}} & x_{I_{1}} & \cdots & x_{I_{w}}\end{array}\right]^{\prime}$ the partition of the vector into the essential and inessential classes.

Consider an essential class, represented by submatrix $T_{i}$. By (3) it follows that

$$
x_{E_{i}}(t)=T_{i}^{t} x_{E_{i}}(0) \quad(i=1, \ldots, q)
$$


and for each primitive (i.e., aperiodic) $T_{i}$, one obtains from Fact 2 the steady-state vector, with $v^{i}$ the left eigenvector of $T_{i}$ such that $v^{i} \cdot \mathbf{1}=1$,

$$
\bar{x}_{E_{i}}=1 v^{\prime i} x(0)
$$

i.e., each agent in $E_{i}$ converges to consensus $v^{i} x(0)$. On the other hand, for inessential agents, the steady-state vector must satisfy

$$
\bar{x}_{I}=R \bar{x}_{E}+Q \bar{x}_{I}
$$

i.e., $\bar{x}_{I}=(I-Q)^{-1} R \bar{x}_{E}$ if $I-Q$ is invertible. By Fact 3, Lemma 1 proves that $I-Q$ is always invertible, so that a steady-state vector $\bar{x}$ always exists, obtained from (6) and (7).

\section{Convergence in the model of influence with two strategic agents}

We consider a set $N=\{1, \ldots, n\}$ of non-strategic agents. Each agent is characterized by an initial opinion (belief) represented by a number in $[0,1]$.

The non-strategic agents are linked through an influence network represented by a directed graph with adjacency matrix $A$. An $\operatorname{arc}$ from $i$ to $j$, i.e., $a_{i j}=1$, means that $i$ listens to the opinion of $j$.

Each agent updates his belief as in the model of DeGroot (1974), i.e., according to a linear combination of his neighbors' beliefs. A simplifying assumption ${ }^{1}$ is to consider that each neighbor is equally weighted:

$$
w_{i j}=\frac{1}{d_{i}} a_{i j}
$$

where $d_{i}=\sum_{j=1}^{n} a_{i j}$ is the out-degree (number of outgoing links) of agent $i$. We call it the uniform (weighting) model. Under this assumption, the weight matrix $W$ is simply $W=D A$, where $D$ is the diagonal matrix with diagonal elements $1 / d_{1}, \ldots, 1 / d_{n}$.

The evolution of the opinion vector $x(t)$ obeys (5), and as explained in Section 3.2, convergence to a consensus occurs in each essential aperiodic class (see (6)), and a steadystate vector exists for agents in inessential classes (see (7)). We give two examples for illustration.

Example 1 Let $N=\{1,2,3\}$ and consider the network given in Figure 1 .

Taking the uniform model, the corresponding matrices are:

$$
A=\left[\begin{array}{lll}
1 & 1 & 0 \\
1 & 1 & 1 \\
1 & 0 & 1
\end{array}\right], D=\left[\begin{array}{ccc}
1 / 2 & 0 & 0 \\
0 & 1 / 3 & 0 \\
0 & 0 & 1 / 2
\end{array}\right], W=D A=\left[\begin{array}{ccc}
1 / 2 & 1 / 2 & 0 \\
1 / 3 & 1 / 3 & 1 / 3 \\
1 / 2 & 0 & 1 / 2
\end{array}\right]
$$

We see that $N$ is the (only) essential class, which is aperiodic, hence $W$ is primitive. Therefore, a consensus $\bar{x}$ is reached in $N$, given by $\bar{x}=\left[\begin{array}{lll}\frac{4}{9} & \frac{1}{3} & \frac{2}{9}\end{array}\right] x(0)$. 


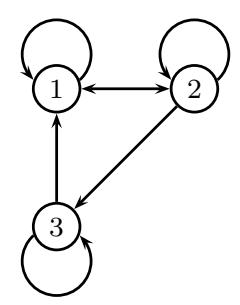

Fig. 1. Example 1: Consensus in $N$

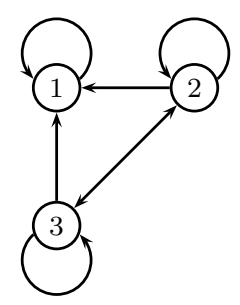

Fig. 2. Example 2: Consensus in $N$

Example 2 Let the network of the non-strategic agents in $N$ be given in Figure 2.

The corresponding matrices are:

$$
A=\left[\begin{array}{lll}
1 & 0 & 0 \\
1 & 1 & 1 \\
1 & 1 & 1
\end{array}\right], D=\left[\begin{array}{ccc}
1 & 0 & 0 \\
0 & 1 / 3 & 0 \\
0 & 0 & 1 / 3
\end{array}\right], W=D A=\left[\begin{array}{ccc}
1 & 0 & 0 \\
1 / 3 & 1 / 3 & 1 / 3 \\
1 / 3 & 1 / 3 & 1 / 3
\end{array}\right]
$$

We see that $\{1\}$ is the only essential class, while $\{2,3\}$ is inessential. Therefore, $W$ is not irreducible. From (6), we immediately have $\bar{x}_{1}=x_{1}(0)$, and from (7) we obtain that $\bar{x}_{2}=\bar{x}_{3}=\bar{x}_{1}$. Hence, also in this case a consensus is reached in $N$.

We then consider two strategic agents $a_{1}$ and $a_{2}$, who have the fixed beliefs 1 and 0 , respectively, and each of them forms exactly one link with a non-strategic agent in order to influence the dynamics of the beliefs. More precisely, each strategic agent $a_{i}$ chooses a strategy $s_{i}$ in $N$ that represents the non-strategic agent he decides to form a link with. We assume throughout the paper that the weights given to agents $a_{1}, a_{2}$ by the agents in $N$ are positive. Our aim is to study the dynamics of beliefs in this modified network.

As before, let $W$ be the $n \times n$ matrix of weights for the agents in $N$, before perturbation by $a_{1}, a_{2}$, and let $P$ be the $(n+2) \times(n+2)$ matrix of weights of the agents in $N$ and $a_{1}$, $a_{2}$. Then, the essential classes of $P$ are $\left\{a_{1}\right\},\left\{a_{2}\right\}$, and all previous classes of $W$ become inessential, say $I_{1}, \ldots, I_{w}$. The structure of $P$, which is still row-stochastic, is therefore

$$
P=\left[\begin{array}{ll|l}
1 & 0 & \mathbf{0} \\
0 & 1 & \mathbf{0} \\
\hline R & Q
\end{array}\right]
$$

where $Q$ has form (2) and $R \neq \mathbf{0}$.

\footnotetext{
${ }^{1}$ This assumption is used to simplify the exposition. Our results can easily be extended to arbitrarily weighted networks.
} 
We may think of specific models for obtaining the weights in $P$ from those in $W$. In particular, we may consider the uniform model introduced above, where $w_{i j}=\frac{a_{i j}}{d_{i}}$, and that the strategic agents $a_{1}, a_{2}$ have an impact of $\lambda$ and $\mu$ respectively $(\lambda, \mu>0)$ when computing the out-degrees. Specifically, if $s_{1}=s_{2}=i$, weight $w_{i j}$ becomes $\frac{1}{d_{i}+\lambda+\mu}$, while weights on $a_{1}, a_{2}$ are respectively $\frac{\lambda}{d_{i}+\lambda+\mu}$ and $\frac{\mu}{d_{i}+\lambda+\mu}$, and if $s_{1} \neq s_{2}$, we get

$$
\begin{aligned}
w_{s_{1} j} & =\frac{a_{i j}}{d_{s_{1}}+\lambda}, \quad j \in N, \\
w_{s_{1} a_{1}} & =\frac{\lambda}{d_{s_{1}}+\lambda}, \quad w_{s_{1} a_{2}}=\frac{\mu}{d_{s_{1}}+\lambda},
\end{aligned}
$$

and similarly for $s_{2}$. Then, one gets

$$
P=\left[\begin{array}{cc|c}
1 & 0 & \mathbf{0} \\
0 & 1 & \mathbf{0} \\
\hline \Delta_{\lambda, \mu}(s) E_{\lambda, \mu}(s) & \Delta_{\lambda, \mu}(s) A
\end{array}\right]
$$

with $E_{\lambda, \mu}(s)=\left[\begin{array}{ll}\lambda e_{s_{1}} & \mu e_{s_{2}}\end{array}\right]$, where $e_{i}$ is the unit vector with coordinate 1 at $i$, and $\Delta_{\lambda, \mu}(s)$ is a diagonal matrix with diagonal elements

$$
\frac{1}{d_{1}+\lambda \delta_{1, s_{1}}+\mu \delta_{1, s_{2}}}, \ldots, \frac{1}{d_{n}+\lambda \delta_{n, s_{1}}+\mu \delta_{n, s_{2}}},
$$

where $\delta$ denotes the Kronecker symbol, i.e., $\delta_{i, s_{j}}=1$ if $i=s_{j}$ and 0 otherwise.

In general, the evolution law of the opinion vector $x(t)$ is given by

$$
x(t+1)=P x(t) .
$$

By writing the opinion vector $x$ as $x=\left[\begin{array}{lll}x_{a_{1}} & x_{a_{2}} & x_{N}\end{array}\right]^{\prime}$, we immediately see that $x_{a_{1}}(t), x_{a_{2}}(t)$ are constant and equal to 1,0 respectively and that the evolution law for the opinions of the non-strategic agents is given by:

$$
x_{N}(t+1)=R\left[\begin{array}{l}
1 \\
0
\end{array}\right]+Q x_{N}(t) .
$$

The convergence of opinions in our model with strategic influence is then a direct consequence of the results recalled in Section 3.2. Namely we have:

Proposition 1 For any initial vector of opinions $x:=\left[\begin{array}{lll}x_{a_{1}} & x_{a_{2}} & x_{N}(0)\end{array}\right]^{\prime}$ with $x_{a_{1}}(0)=1$, $x_{a_{2}}(0)=0$ and $x_{N}(0) \in[0,1]^{n}$, we have:

$$
\lim _{t \rightarrow+\infty} P^{t}\left[\begin{array}{lll}
x_{a_{1}} & x_{a_{2}} & x_{N}(0)
\end{array}\right]^{\prime}=\left[\begin{array}{lll}
\bar{x}_{a_{1}} & \bar{x}_{a_{2}} & \bar{x}_{N}
\end{array}\right]^{\prime}
$$

where $\bar{x}_{a_{1}}=1, \bar{x}_{a_{2}}=0$ and $\bar{x}_{N}=(I-Q)^{-1} R[1 \quad 0]^{\prime}$.

In particular, if the two strategic agents choose the same target, one obtains convergence towards a consensus.

Proposition 2 Let $P$ be the $(n+2) \times(n+2)$ matrix of weights obtained if $a_{1}, a_{2}$ target the same agent $i$, and only this one. Then agents in $N$ reach a consensus $\alpha$, given by $\alpha=\frac{\alpha_{1}}{\alpha_{1}+\alpha_{2}}$, where $\alpha_{1}, \alpha_{2}$ are the weights put on agents $a_{1}, a_{2}$ by agent $i$. 
Proof: Suppose that $a_{1}, a_{2}$ target $i$. Then

$$
R=\left[\begin{array}{cc}
0 & 0 \\
\vdots & \vdots \\
\alpha_{1} & \alpha_{2} \\
\vdots & \vdots \\
0 & 0
\end{array}\right]
$$

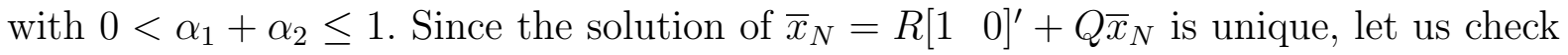
if a consensus vector $\bar{x}_{N}=\left[\begin{array}{lll}\alpha & \cdots & \alpha\end{array}\right]^{\prime}$ is a solution. We have for all rows $j \neq i$ :

$$
\alpha=0+1 \cdot \alpha=\alpha
$$

since $P$ is row-stochastic, and for row $i$, we obtain:

$$
\alpha=\alpha_{1}+\left(1-\alpha_{1}-\alpha_{2}\right) \alpha
$$

which yields $\alpha=\frac{\alpha_{1}}{\alpha_{1}+\alpha_{2}}$.

\section{Nash equilibrium in the model with two strategic agents}

\subsection{Convergence and consensus reaching}

In the remaining of the paper we place ourselves in the "uniform" model where the dynamics of opinions are given by equation (8). In this setting, Proposition 1 implies convergence of the beliefs towards the steady-state vector $\bar{x}_{N} \in[0,1]^{n}$ such that

$$
\bar{x}_{N}=\left(I-\Delta_{\lambda, \mu}(s) A\right)^{-1} \lambda \Delta_{\lambda, \mu}(s) e_{s_{1}} .
$$

Note that this steady state vector is independent of the initial beliefs of the non-strategic agents, but of course depends on the strategy vector $s$.

Moreover, according to Proposition 2, in the case where both strategic agents choose the same target, one obtains convergence towards a consensus equal to $\alpha=\frac{\lambda}{\lambda+\mu}$. In particular, if the weights are equal, the consensus is $1 / 2$.

Example 3 Let us illustrate these results for the network of non-strategic agents presented in Figure 2. We set $\lambda=\mu=1$ and consider the following cases:

Case 1: $s_{1}=s_{2}=1$. Then

$$
\left[\begin{array}{ll}
R & Q
\end{array}\right]=\left[\begin{array}{ccccc}
1 / 3 & 1 / 3 & 1 / 3 & 0 & 0 \\
0 & 0 & 1 / 3 & 1 / 3 & 1 / 3 \\
0 & 0 & 1 / 3 & 1 / 3 & 1 / 3
\end{array}\right]
$$

The solution of $\bar{x}_{N}=R\left[\begin{array}{ll}1 & 0\end{array}\right]^{\prime}+Q \bar{x}_{N}$ is $\bar{x}_{1}=\bar{x}_{2}=\bar{x}_{3}=1 / 2$. 
Case 2: $s_{1}=s_{2}=2$. Then

$$
\left[\begin{array}{ll}
R & Q
\end{array}\right]=\left[\begin{array}{ccccc}
0 & 0 & 1 & 0 & 0 \\
1 / 5 & 1 / 5 & 1 / 5 & 1 / 5 & 1 / 5 \\
0 & 0 & 1 / 3 & 1 / 3 & 1 / 3
\end{array}\right]
$$

The solution is $\bar{x}_{1}=x_{1}(0)$, and $\bar{x}_{2}=\frac{3}{7} x_{1}(0), \bar{x}_{3}=\frac{5}{7} x_{1}(0)$.

Case 3: $s_{1}=1, s_{2}=2$. Then

$$
\left[\begin{array}{ll}
R & Q
\end{array}\right]=\left[\begin{array}{ccccc}
1 / 2 & 0 & 1 / 2 & 0 & 0 \\
0 & 1 / 4 & 1 / 4 & 1 / 4 & 1 / 4 \\
0 & 0 & 1 / 3 & 1 / 3 & 1 / 3
\end{array}\right]
$$

The solution of $\bar{x}_{N}=R[1 \quad 0]^{\prime}+Q \bar{x}_{N}$ is $\bar{x}_{1}=1, \bar{x}_{2}=3 / 5, \bar{x}_{3}=4 / 5$.

Case $4: s_{1}=2, s_{2}=1$. Then

$$
\left[\begin{array}{ll}
R & Q
\end{array}\right]=\left[\begin{array}{ccccc}
0 & 1 / 2 & 1 / 2 & 0 & 0 \\
1 / 4 & 0 & 1 / 4 & 1 / 4 & 1 / 4 \\
0 & 0 & 1 / 3 & 1 / 3 & 1 / 3
\end{array}\right]
$$

The solution of $\bar{x}_{N}=R\left[\begin{array}{ll}1 & 0\end{array}\right]^{\prime}+Q \bar{x}_{N}$ is $\bar{x}_{1}=0, \bar{x}_{2}=2 / 5, \bar{x}_{3}=1 / 5$.

\subsection{Payoffs and best responses of the strategic agents}

We now turn to the core of our analysis and consider the zero-sum game $\mathcal{G}_{\lambda, \mu}$ with players $a_{1}, a_{2}$ whose respective impacts are $\lambda, \mu>0$ and whose sets of strategies are $s=\left(s_{1}, s_{2}\right) \in N \times N$. We assume that the payoff of each strategic agent depends on the average asymptotic belief. That is, the payoff $\pi_{\lambda, \mu}(s)$ of player $a_{1}$ is given by

$$
\pi_{\lambda, \mu}(s)=\mathbf{1}^{\prime} \cdot\left(I-\Delta_{\lambda, \mu}(s) A\right)^{-1} \lambda \Delta_{\lambda, \mu}(s) e_{s_{1}}
$$

and player $a_{2}$ 's payoff is equal to $n-\pi_{\lambda, \mu}(s)$. We know from Section 5.1 that $I-\Delta(s) A$ is indeed invertible and that the payoff can also be written as

$$
\pi_{\lambda, \mu}(s)=\mathbf{1}^{\prime} \cdot \sum_{m=0}^{\infty}\left(\Delta_{\lambda, \mu}(s) A\right)^{m} \lambda \Delta_{\lambda, \mu}(s) e_{s_{1}}
$$

Example 4 In Example 3, we have the following:

- for $s_{1}=s_{2}=1, \bar{x}=\left[\frac{1}{2}, \frac{1}{2}, \frac{1}{2}\right]$, the payoff of each strategic agent is $3 / 2$;

- for $s_{1}=1, s_{2}=2, \bar{x}=\left[1, \frac{3}{5}, \frac{4}{5}\right]$, the payoff of agent $a_{1}$ is $12 / 5$ and the payoff of agent $a_{2}$ is $3 / 5$;

- for $s_{1}=2, s_{2}=1, \bar{x}=\left[0, \frac{2}{5}, \frac{1}{5}\right]$, the payoffs of agents $a_{1}$ and $a_{2}$ are $3 / 5$ and $12 / 5$, respectively. 
The game $\mathcal{G}_{\lambda, \mu}$ being a zero-sum game with finite strategy space, it definitely has at least an equilibrium in mixed strategies. Yet mixed strategies have little relevance as prescriptions for link formation. We thus proceed with the characterization of the conditions under which the game admits equilibria in pure strategies. We place ourselves in the following in the case where the network between non-strategic agents is simply connected and therefore the matrix $W$ is irreducible.

We shall derive a more explicit expression of the payoff function. Let us then denote by $\mathcal{P}_{k, l}^{m}$ the set of walks of length $m$ from $k$ to $l$. For any walk $p=\left(i_{1}, \cdots, i_{m}\right)$ we denote by $w(p)$ its "weight" measured according to $W=D A$, i.e.,

$$
w(p):=\prod_{j=1}^{m-1} w_{i_{j}, i_{j+1}}
$$

We also denote by $\nu_{k}(p)$ the number of times the walk $p$ passes through $k$ (without taking into account the departure node). Equation (13) can then be rewritten as a sum representing the influence conveyed through each walk of the networks and where each passage through one of the target node is re-weighted in order to account for the influence of the external agents. That is, one has:

- if $s_{1}=s_{2}$ then

$$
\pi_{\lambda, \mu}(s)=\left(\sum_{k \in N} \sum_{m=0}^{\infty} \sum_{p \in \mathcal{P}_{k, s_{1}}^{m}} w(p)\left(\frac{d_{s_{1}}}{d_{s_{1}}+\lambda+\mu}\right)^{\nu_{s_{1}}(p)}\right) \frac{\lambda}{d_{s_{1}}+\lambda+\mu}
$$

- if $s_{1} \neq s_{2}$ then

$$
\pi_{\lambda, \mu}(s)=\left(\sum_{k \in N} \sum_{m=0}^{\infty} \sum_{p \in \mathcal{P}_{k, s_{1}}^{m}} w(p)\left(\frac{d_{s_{1}}}{d_{s_{1}}+\lambda}\right)^{\nu_{s_{1}}(p)}\left(\frac{d_{s_{2}}}{d_{s_{2}}+\mu}\right)^{\nu_{s_{2}}(p)}\right) \frac{\lambda}{d_{s_{1}}+\lambda}
$$

It is already clear from this expression that the payoff of a link to agent $i$ depends on the influenceability of the agent (measured by the number of outgoing links of the agent) and the influence of the agent measured by the (weighted and discounted) number of walks that pass through that agent. This formula also allows to further characterize the payoff in some particular cases of interest.

Remark 1 If all the non-strategic agents have the same out-degree d, the payoff is given by:

$$
\begin{aligned}
& \pi_{\lambda, \mu}(s)=\left(\sum_{k \in N} \sum_{m=0}^{+\infty} \sum_{p \in P_{k, s_{1}}^{m}}\left(\frac{1}{d}\right)^{m}\left(\frac{d}{d+\lambda+\mu}\right)^{\nu_{s_{1}}(p)}\right) \frac{\lambda}{d+\lambda+\mu} \quad \text { if } s_{1}=s_{2} \\
& \pi_{\lambda, \mu}(s)=\left(\sum_{k \in N} \sum_{m=0}^{+\infty} \sum_{p \in P_{k, s_{1}}^{m}}\left(\frac{1}{d}\right)^{m} \frac{d^{\nu_{s_{1}}(p)+\nu_{s_{2}}(p)}}{(d+\lambda)^{\nu_{s_{1}}(p)}(d+\mu)^{\nu_{s_{2}}(p)}}\right) \frac{\lambda}{d+\lambda} \quad \text { if } s_{1} \neq s_{2}
\end{aligned}
$$


In particular, for $d=1$, we have:

$$
\begin{gathered}
\pi_{\lambda, \mu}(s)=\sum_{k \in N} \sum_{m=0}^{+\infty} \sum_{p \in P_{k, s_{1}}^{m}} \frac{\lambda}{(1+\lambda+\mu)^{\nu_{s_{1}}(p)+1}} \quad \text { if } s_{1}=s_{2} \\
\pi_{\lambda, \mu}(s)=\sum_{k \in N} \sum_{m=0}^{+\infty} \sum_{p \in P_{k, s_{1}}^{m}} \frac{\lambda}{(1+\lambda)^{\nu_{s_{1}}(p)+1}(1+\mu)^{\nu_{s_{2}}(p)}} \quad \text { if } s_{1} \neq s_{2}
\end{gathered}
$$

If we additionally assume that the network is a circle, then a walk $p$ of length $m$ from $k$ to $s_{1}$ passes $m \div n$ times ${ }^{2}$ through any node plus once in every node between $k$ and $s_{1}$. Let $\sigma\left(s_{2}, s_{1}\right)$ denote the length of the shortest walk between $s_{2}$ and $s_{1}\left(\operatorname{setting} \sigma\left(s_{1}, s_{1}\right)=0\right)$. Then one has:

- if $s_{1}=s_{2}$ then

$$
\pi_{\lambda, \mu}(s)=n \sum_{h=0}^{+\infty} \frac{\lambda}{(1+\lambda+\mu)^{h+1}}=\frac{n \lambda}{\lambda+\mu}
$$

- if $s_{1} \neq s_{2}$ then

$$
\pi_{\lambda, \mu}(s)=\sum_{h=0}^{+\infty}\left[\frac{\sigma\left(s_{2}, s_{1}\right) \lambda}{(1+\lambda)^{h+1}(1+\mu)^{h}}+\frac{\left(n-\sigma\left(s_{2}, s_{1}\right)\right) \lambda}{(1+\lambda)^{h+1}(1+\mu)^{h+1}}\right]=\frac{\lambda\left(n+\mu \sigma\left(s_{2}, s_{1}\right)\right)}{\lambda+\mu+\lambda \mu}
$$

Hence, if we focus on the best-response of player $a_{2}$, playing $s_{2}$ such that $\sigma\left(s_{2}, s_{1}\right)=1$ is better for player $a_{2}$ than playing any other strategy different from $s_{1}$. In other words, the best strategy for agent $a_{2}$ is to target the non-strategic agent immediately before $s_{1}$ on the circle. The payoff is then equal to $\pi_{\lambda, \mu}(s)=\frac{\lambda(n+\mu)}{\lambda+\mu+\lambda \mu}$. Also note that (20) coincides with the result given by Proposition 2.

In order to compute the payoff function for an arbitrary network, we introduce the following auxiliary concepts. We denote by $\Gamma_{i}$ the set of cycles around $i$ (i.e., walks that start and finish in $i$ ) that do not pass through $i$ in between and let $\gamma_{i}=\sum_{p \in \Gamma_{i}} w(p)$. We denote by $\Phi_{i}$ the set of walks to $i$ that have never passed through $i$ before and let $\phi_{i}:=\sum_{p \in \Phi_{i}} w(p)$.

First, we consider symmetric strategies. Assume that both players play $i$ and let $\Pi_{i}^{k}$ denote the set of walks that end in $i$ and have gone exactly $k$ times through $i$ before. Then one has:

$$
\pi_{\lambda, \mu}(i, i)=\left(\sum_{k=0}^{+\infty} \sum_{p \in \Pi_{i}^{k}} w(p)\left(\frac{d_{i}}{d_{i}+\lambda+\mu}\right)^{k}\right) \frac{\lambda}{d_{i}+\lambda+\mu}
$$

A walk in $\Pi_{i}^{k}$ consists in $k$ cycles around $i$ plus, possibly, a walk to $i$. Hence,

$$
\sum_{p \in \Pi_{i}^{k}} w(p)=\left(\gamma_{i}\right)^{k}\left(1+\phi_{i}\right)
$$

\footnotetext{
${ }^{2}$ We denote by $m \div n$ the quotient of the Euclidian division of $m$ by $n$.
} 
and inserting (23) into (22) leads to

$$
\pi_{\lambda, \mu}(i, i)=\left(\sum_{k=0}^{+\infty}\left(\gamma_{i} \frac{d_{i}}{d_{i}+\lambda+\mu}\right)^{k}\left(1+\phi_{i}\right)\right) \frac{\lambda}{d_{i}+\lambda+\mu}=\frac{\lambda\left(1+\phi_{i}\right)}{d_{i}+\lambda+\mu-\gamma_{i} d_{i}}
$$

Hence, the payoff under symmetric strategies is

$$
\pi_{\lambda, \mu}(i, i)=\frac{\lambda\left(1+\phi_{i}\right)}{d_{i}+\lambda+\mu-\gamma_{i} d_{i}}
$$

We have the following:

Lemma 2 If $W$ is irreducible, then for all $i=1, \ldots, n, \gamma_{i}=1$ and $\phi_{i}=n-1$. Consequently, $\pi_{\lambda, \mu}(i, i)=\frac{n \lambda}{\lambda+\mu}$.

Proof: Take $i \in N$ and compute $\gamma_{i}$, i.e., the sum of all weights of cycles starting at $i$, without passing through $i$ twice. We remark that the weight of a walk from $i$ to $j$ is the probability of going from $i$ to $j$ ( $i, j$ being seen as states) using exactly the sequence of states in the walk. Hence, $\gamma_{i}$ is the probability to start from $i$ and to return to $i$ by some walk. Since $W$ is irreducible, this probability is equal to 1.

Similarly, consider $\phi_{i}$, i.e., the sum of all weights of walks that arrive to $i$ without passing through $i$ before. Any such walk must start from some node $j \neq i$. Again, since $W$ is irreducible, the probability to start from $j$ and arriving at $i$ is equal to 1 , and therefore $\phi_{i}=n-1$. Inserting $\gamma_{i}=1$ and $\phi_{i}=n-1$ in $(24)$ yields $\pi_{\lambda, \mu}(i, i)=\frac{n \lambda}{\lambda+\mu}$.

Note that the value of $\pi_{\lambda, \mu}(i, i)$ stated in Lemma 2 was already obtained in Proposition 2 .

Next, we consider the case where player $a_{1}$ plays $i$ and player $a_{2}$ plays $j$, i.e., we assume that $s_{1}=i$ and $s_{2}=j$. We denote by $\mathcal{C}_{i}^{j}$ the set of cycles around $i$ that do not pass through $j$ and by $\mathcal{B}_{i}^{j}$ the set of walks to $i$ that do not pass through $j$. Let $\mathcal{F}_{i, j}$ denote the set of direct walks from $i$ to $j$, i.e., the set of walks that start in $i$, end up in $j$ and do not pass through $i$ nor $j$ in between. We also let

$$
c_{i}^{j}:=\sum_{p \in \mathcal{C}_{i}^{j}} w(p), \quad b_{i}^{j}:=\sum_{p \in \mathcal{B}_{i}^{j}} w(p), \quad f_{i, j}:=\sum_{p \in \mathcal{F}_{i, j}} w(p)
$$

The corresponding set of walks and measures for $j$ are denoted by $\mathcal{C}_{j}^{i}, \mathcal{B}_{j}^{i}, \mathcal{F}_{j, i}, c_{j}^{i}, b_{j}^{i}$, and $f_{j, i}$. We have the following:

Lemma 3 If $W$ is irreducible, then for all $i, j \in N$ and $i \neq j, b_{i}^{j}+b_{j}^{i}=n-2$.

Proof: We proceed as in Lemma 2, interpreting weights of walks as probabilities. Let $i, j$ be fixed and consider walks from a node $k \neq i, j$ to $i$. As $W$ is irreducible, the probability of going from $k$ to $i$ is equal to 1 . Observe that a walk from $k$ to $i$ either passes through $j$ or not, and that in the former case, a walk from $k$ to $i$ is decomposed into a walk from $k$ to $j$ without passing through $i$ and a walk from $j$ to $i$. Thus we obtain, after factorization:

$$
1=\sum_{\substack{p \in \mathcal{B}_{i}^{j} \\ p \text { starts from } k}} w(p)+\sum_{\substack{p \in \mathcal{B}_{j}^{i} \\ p \text { starts from } k}} w(p) \sum_{p^{\prime} \in \mathcal{F}_{j, i}} w\left(p^{\prime}\right)
$$


Observe that $\sum_{p^{\prime} \in \mathcal{F}_{j, i}} w\left(p^{\prime}\right)=1$. It follows that, summing over all $k \neq i, j$ :

$$
\begin{aligned}
n-2= & \sum_{k \neq i, j} \sum_{\substack{p \in \mathcal{B}_{i}^{j} \\
p \text { starts from } k}} w(p)+\sum_{k \neq i, j} \sum_{\substack{p \in \mathcal{B}_{j}^{i} \\
p \text { starts from } k}} w(p) \\
& =b_{i}^{j}+b_{j}^{i} .
\end{aligned}
$$

Let us denote by $\phi_{i}^{j}$ the sum of weights of the walks to $i$ but where each passage through $j$ is weighted by the factor $\frac{d_{j}}{d_{j}+\mu}$. Similarly, let us denote by $\gamma_{i}^{j}$ the sum of weights of walks that cycle around $i$ but where each passage through $j$ is weighted by the factor $\frac{d_{j}}{d_{j}+\mu}$. We have:

$$
\phi_{i}^{j}=b_{i}^{j}+f_{j, i} \frac{d_{j}}{d_{j}+\mu}\left(\sum_{k=0}^{+\infty}\left(c_{j}^{i} \frac{d_{j}}{d_{j}+\mu}\right)^{k}\right)\left(1+b_{j}^{i}\right)=b_{i}^{j}+f_{j, i} \frac{d_{j}}{d_{j}+\mu-c_{j}^{i} d_{j}}\left(1+b_{j}^{i}\right)
$$

because the set of walks to $i$ consists in walks to $i$ that do not pass through $j$ plus the set of direct walks from $j$ to $i$ preceded by an arbitrary number of cycles around $j$ (not passing through $i$ ) preceded by a walk to $j$ that do not pass through $i$.

In a similar fashion

$$
\gamma_{i}^{j}=c_{i}^{j}+f_{j, i} \frac{d_{j}}{d_{j}+\mu}\left(\sum_{k=0}^{+\infty}\left(c_{j}^{i} \frac{d_{j}}{d_{j}+\mu}\right)^{k}\right) f_{i, j}=c_{i}^{j}+f_{i, j} f_{j, i} \frac{d_{j}}{d_{j}+\mu-c_{j}^{i} d_{j}}
$$

because the set of cycles around $i$ consists in the set of cycles around $i$ not passing through $j$ together with the set of direct walks from $j$ to $i$ preceded by an arbitrary number of cycles around $j$ (not passing through $i$ ) preceded by a direct walk from $i$ to $j$.

An argument similar to the one in the symmetric case yields:

$$
\pi_{\lambda, \mu}(i, j)=\frac{\lambda\left(1+\phi_{i}^{j}\right)}{d_{i}+\lambda-\gamma_{i}^{j} d_{i}}
$$

Using equations (25) and (26) we get:

$$
\begin{aligned}
\pi_{\lambda, \mu}(i, j) & =\frac{\lambda\left(1+b_{i}^{j}+f_{j, i} \frac{d_{j}}{d_{j}+\mu-c_{j}^{i} d_{j}}\left(1+b_{j}^{i}\right)\right)}{d_{i}+\lambda-\left(c_{i}^{j}+f_{i, j} f_{j, i} \frac{d_{j}}{d_{j}+\mu-c_{j}^{i} d_{j}}\right) d_{i}} \\
= & \frac{\lambda\left[\left(1+b_{i}^{j}\right) \mu+d_{j}\left(1-c_{j}^{i}\right)\left(1+b_{i}^{j}+f_{j, i} \frac{1+b_{j}^{i}}{1-c_{j}^{i}}\right)\right]}{\left(d_{i}+\lambda\right)\left(d_{j}+\mu-c_{j}^{i} d_{j}\right)-d_{i}\left(c_{i}^{j} \mu+d_{j} c_{i}^{j}\left(1-c_{j}^{i}\right)+d_{j} f_{i, j} f_{j, i}\right)}
\end{aligned}
$$

One can then remark (when $\gamma_{i}$ and $\phi_{i}$ are defined as in the symmetric case, i.e., without further weighting when passing through $j$ ) that

$$
\phi_{i}=b_{i}^{j}+f_{j, i}\left(\sum_{k=0}^{+\infty}\left(c_{j}^{i}\right)^{k}\right)\left(1+b_{j}^{i}\right)=b_{i}^{j}+f_{j, i} \frac{1}{1-c_{j}^{i}}\left(1+b_{j}^{i}\right)
$$




$$
\gamma_{i}=c_{i}^{j}+f_{j, i}\left(\sum_{k=0}^{+\infty}\left(c_{j}^{i}\right)^{k}\right) f_{i, j}=c_{i}^{j}+f_{i, j} f_{j, i} \frac{1}{1-c_{j}^{i}}
$$

Hence, we have:

$$
\pi_{\lambda, \mu}(i, j)=\frac{\lambda\left[\mu\left(1+b_{i}^{j}\right)+d_{j}\left(1-c_{j}^{i}\right)\left(1+\phi_{i}\right)\right]}{\left(d_{i}+\lambda\right)\left(d_{j}+\mu-c_{j}^{i} d_{j}\right)-d_{i}\left(c_{i}^{j} \mu+d_{j}\left(1-c_{j}^{i}\right) \gamma_{i}\right)}
$$

Using similar arguments as in Lemma 2, we then get:

Lemma 4 If $s_{1}=i$ and $s_{2}=j \neq i$, one has:

$$
\pi_{\lambda, \mu}(i, j)=\frac{\lambda\left[\mu\left(1+b_{i}^{j}\right)+d_{j}\left(1-c_{j}^{i}\right) n\right]}{\mu d_{i}\left(1-c_{i}^{j}\right)+\lambda d_{j}\left(1-c_{j}^{i}\right)+\lambda \mu}
$$

Note that one necessarily has $c_{i}^{j}<1$, because the network is simply connected and hence at least one cycle around $i$ passes through $j$. Similarly, one has $c_{j}^{i}<1, b_{i}^{j}<n-1$ and $b_{j}^{i}<n-1$.

\subsection{Characterizing equilibria - equal influence}

We first place ourselves in a setting where both strategic agents exert the same impact, i.e., $\lambda=\mu$. In this setting, the structure of the game is perfectly symmetric and so shall be the payoff functions (as a matter of fact if $\lambda=\mu$, we slightly abuse the notation and write $\pi_{\lambda}$ instead of $\pi_{\lambda, \lambda}$ ). This fact is not clear from equation (13) but it is a direct consequence of Lemmas 2 to 4 . Indeed, one then has:

Proposition 3 If $\lambda=\mu$, then one has for all $i, j \in N$ :

$$
\pi_{\lambda}(i, j)+\pi_{\lambda}(j, i)=n
$$

Proof: From equation (31) and Lemma 2, we have:

$$
\pi_{\lambda}(i, j)=\frac{\lambda\left(1+b_{i}^{j}\right)+d_{j}\left(1-c_{j}^{i}\right) n}{d_{i}\left(1-c_{i}^{j}\right)+d_{j}\left(1-c_{j}^{i}\right)+\lambda}, \quad \pi_{\lambda}(i, i)=\frac{n}{2}
$$

and from Lemma 3, we get:

$$
\pi_{\lambda}(i, j)+\pi_{\lambda}(j, i)=n
$$

Hence, the game is symmetric and standard results about symmetric zero-sum games (see Duersch et al. (2012)) imply the following characterization of equilibria in pure strategies (we write $\mathcal{G}_{\lambda}$ instead of $\mathcal{G}_{\lambda, \lambda}$ ):

Theorem 1 (i) A pair of strategies $(i, i)$ is an equilibrium of the game $\mathcal{G}_{\lambda}$ if for all $j \in N$ :

$$
\lambda\left[2\left(1+b_{i}^{j}\right)-n\right] \geq n\left[d_{i}\left(1-c_{i}^{j}\right)-d_{j}\left(1-c_{j}^{i}\right)\right]
$$


(ii) If a pair of strategies $(i, j)$ is an equilibrium of the game $\mathcal{G}_{\lambda}$, then also $(i, i)$ and $(j, j)$ are equilibria.

Proof: Given the game is symmetric, $(i, i)$ is an equilibrium if and only if player $a_{2}$ has no profitable deviation, that is for all $j \in N, \pi_{\lambda}(i, j) \geq \pi_{\lambda}(i, i)$. Part $(i)$ of the theorem then follows from equation (33). Part (ii) is a direct consequence of the fact that the game is symmetric.

A first consequence of Theorem 1 is that we are able to show that there indeed exist influence networks that admit equilibria in pure strategies as underlined in the following remark.

Remark 2 Let us consider a perfectly symmetric network, i.e., a network such that for all $i, j \in N, d_{i}=d_{j}, c_{i}^{j}=c_{j}^{i}, b_{i}^{j}=b_{j}^{i}$, and $f_{i, j}=f_{j, i}$. Then condition (35) always holds so that every $(i, i)$ is an equilibrium of the game $\mathcal{G}_{\lambda}$ in this network.

Hence, combining Remarks 1 and 2, it appears that the network structure indeed has implications for the existence of an equilibrium in pure strategies.

Conclusion 1 There are networks for which there exist equilibria in pure strategies (e.g., perfectly symmetric networks) as well as networks for which there exists no equilibrium in pure strategies (e.g., the circle).

A second consequence of Theorem 1 is that symmetric equilibria shall be the focal points of our analysis. In order to proceed with the characterization of these symmetric equilibria, we first consider the two polar cases where the impact of the strategic agents is either infinitely large or infinitely small with respect to the normalized influence within the network. In this respect, one has:

\section{Proposition 4}

(i) One has $\lim _{\lambda \rightarrow 0} \pi_{\lambda}(i, j)=\frac{d_{j}\left(1-c_{j}^{i}\right) n}{d_{i}\left(1-c_{i}^{j}\right)+d_{j}\left(1-c_{j}^{i}\right)}$, so that $(i, i)$ is an equilibrium of the game $\mathcal{G}_{\lambda}$ as $\lambda \rightarrow 0$ if and only if for all $j \in N$ :

$$
d_{j}\left(1-c_{j}^{i}\right) \geq d_{i}\left(1-c_{i}^{j}\right)
$$

(ii) One has $\lim _{\lambda \rightarrow+\infty} \pi_{\lambda}(i, j)=1+b_{i}^{j}$, so that $(i, i)$ is an equilibrium of the game $\mathcal{G}_{\lambda}$ as $\lambda \rightarrow+\infty$ if and only if for all $j \in N$ :

$$
b_{i}^{j} \geq b_{j}^{i}
$$

As mentioned above, one necessarily has $0<c_{i}^{j}<1$ for a simply connected network, so that condition $(i)$ in Proposition 4 can equivalently be written as:

$$
\frac{1}{d_{j}\left(1-c_{j}^{i}\right)} \leq \frac{1}{d_{i}\left(1-c_{i}^{j}\right)}
$$


The quantity $\frac{1}{d_{i}\left(1-c_{i}^{j}\right)}$ is a measure of influenceability of agent $i$ corrected by the external influence exerted on the network. Indeed, it corresponds to the default influenceability, $\frac{1}{d_{i}}$, amplified by a factor $\frac{1}{1-c_{i}^{j}}$, that accounts for the self-feedback that $i$ exerts on itself through the network (i.e., the cycles around $i$ ) without interference from $j$ (i.e., the cycles not containing $j$ ). Hence, if $c_{i}^{j}=0$, that is, if all the cycles around $i$ pass through $j$, then $i$ has only a default influenceability of $\frac{1}{d_{i}}$. On the contrary, as $c_{i}^{j}$ tends towards one, that is, as $i$ is shielded away from the influence of $j$, then the influenceability of $i$ tends towards infinity as agent $i$ will eventually be exposed only to the external influence he receives. We can then interpret the first part of Proposition 4 as follows: $(i, i)$ is an equilibrium for a vanishingly small level of influence only if $i$ is relatively more influenceable than any other agent $j \in N$.

To clarify the interpretation of the other polar case where the level of influence tends towards infinity, it is useful to introduce the quantity $\bar{b}_{i}^{j}$ to denote the sum of weights of the walks to $i$ passing through $j$. Then by virtue of Lemma 2 one has that $b_{i}^{j}+\bar{b}_{i}^{j}=n-1$, so that $\pi(i, j)=n-\bar{b}_{i}^{j}$ and condition (ii) of Proposition 4 is equivalent to

$$
\bar{b}_{j}^{i} \geq \bar{b}_{i}^{j}
$$

This quantity $\bar{b}_{j}^{i}$ can naturally be interpreted as a measure of the influence of $j$ on $i$. Hence, one can interpret the second part of Proposition 4 as follows: in the case where $\lambda \rightarrow+\infty,(i, i)$ is a Nash equilibrium if and only if for all $j$, the relative influence of $j$ on $i$ is greater than the relative influence of $i$ on $j$ as measured by $\bar{b}_{j}^{i}$ and $\bar{b}_{i}^{j}$, respectively.

Furthermore, as $\pi_{\lambda}(i, j)+\pi_{\lambda}(j, i)=n$, and hence $\bar{b}_{i}^{j}+\bar{b}_{j}^{i}=n$, equation (39) is also equivalent to $\bar{b}_{j}^{i} \geq \frac{n}{2}$. Therefore it seems natural to introduce as an absolute measure of influence in our context the quantity $\bar{B}_{i}=\min _{j \neq i} \bar{b}_{j}^{i}$ representing the minimal number of walks to a given node going through $i$. In this sense $\bar{B}_{i}$ measures the "intermediacy" of agent $i$. It is then clear that $(i, i)$ is an equilibrium if and only if $\bar{B}_{i} \geq \frac{n}{2}$.

We now consider the case of an arbitrary $\lambda>0$. According to equation $(35),(i, i)$ is an equilibrium if and only if:

$$
\lambda\left[2\left(1+b_{i}^{j}\right)-n\right] \geq n\left[d_{i}\left(1-c_{i}^{j}\right)-d_{j}\left(1-c_{j}^{i}\right)\right]
$$

Using the fact that $b_{i}^{j}=n-1-\bar{b}_{i}^{j}$, this is equivalent to:

$$
\lambda\left[n-2 \bar{b}_{i}^{j}\right] \geq n\left[d_{i}\left(1-c_{i}^{j}\right)-d_{j}\left(1-c_{j}^{i}\right)\right]
$$

As moreover $n=\bar{b}_{i}^{j}+\bar{b}_{j}^{i}$, this yields:

$$
\bar{b}_{j}^{i}-\bar{b}_{i}^{j} \geq \frac{n}{\lambda}\left[d_{i}\left(1-c_{i}^{j}\right)-d_{j}\left(1-c_{j}^{i}\right)\right]
$$

Hence, in the general case, the equilibrium condition depends both on the influence and the influenceability of the target $i$. Namely $(i, i)$ is an equilibrium if for all $j \in N$, the 
excess influence/intermediacy of $j$ over $i$ is greater than the excess influenceability of $j$ over $i$, scaled by the factor $\frac{n}{\lambda}$. As we have seen above, the relative importance of influence vis-à-vis influenceability increases with the level of external influence $\lambda$. Conversely, as the size of the network increases, the relative importance of influence vis-à-vis influenceability decreases. In other words, as the size of the network grows, highly influenceable agents become optimal targets.

\subsection{Characterizing equilibria - unequal influence}

We now turn back to the general case where $\lambda \neq \mu$ and assume, without loss of generality, that $\lambda>\mu$. As the game is no longer symmetric, $(i, i)$ no longer is a natural candidate for equilibrium in pure strategies. As a matter of fact, using equation (31), one has $\pi_{\lambda, \mu}(i, j) \geq \pi_{\lambda, \mu}(i, i)$ for some $j \neq i$, only if

$$
(\lambda+\mu)\left[\mu\left(1+b_{i}^{j}\right)+n d_{j}\left(1-c_{j}^{i}\right)\right] \geq n\left[\mu d_{i}\left(1-c_{i}^{j}\right)+\lambda d_{j}\left(1-c_{j}^{i}\right)+\lambda \mu\right]
$$

Using Lemma 3, i.e., the fact that $\left(1+b_{i}^{j}\right)+\left(1+b_{j}^{i}\right)=n$, this simplifies to:

$$
\mu\left(1+b_{i}^{j}\right)-\lambda\left(1+b_{j}^{i}\right) \geq n\left[d_{i}\left(1-c_{i}^{j}\right)-d_{j}\left(1-c_{j}^{i}\right)\right]
$$

Using the fact that $1+b_{i}^{j}=n-\bar{b}_{i}^{j}$ and $\bar{b}_{i}^{j}+\bar{b}_{j}^{i}=n$, this eventually yields

$$
(\mu+\lambda) \bar{b}_{j}^{i}-\lambda n \geq n\left[d_{i}\left(1-c_{i}^{j}\right)-d_{j}\left(1-c_{j}^{i}\right)\right]
$$

Similarly, one obtains that $\pi_{\lambda, \mu}(i, i) \geq \pi_{\lambda, \mu}(j, i)$ for some $j \neq i$, only if

$$
(\mu+\lambda) \bar{b}_{j}^{i}-\mu n \geq n\left[d_{i}\left(1-c_{i}^{j}\right)-d_{j}\left(1-c_{j}^{i}\right)\right]
$$

Hence, as $\lambda>\mu$, a necessary and sufficient condition for $(i, i)$ to be an equilibrium is that equation (44) holds true for all $j \neq i$. Now, it is clear that unless $\bar{b}_{j}^{i}=n$, that is unless all the walks to $j$ go through $i$, one has $\bar{b}_{j}^{i}<n$ and hence the left hand side of equation (44) tends towards $-\infty$ as $\lambda$ tends towards $+\infty$. This implies that for $\lambda$ large enough (and a given $\mu$ ), equation (44) cannot hold and hence $(i, i)$ cannot be a Nash equilibrium. Namely, one has:

Proposition 5 For fixed $\mu>0$ and large enough $\lambda$, there exists no $i \in N$ such that $(i, i)$ is an equilibrium in pure strategy of $\mathcal{G}_{\lambda, \mu}$.

The interpretation of this result is relatively straightforward: if one of the strategic agents has a much larger level of influence than the other one, then the low influence agent should not choose the same target as the high influence agent because his influence would get washed away.

Let us then consider a pair of strategies $(i, j) \in N \times N$. It is an equilibrium if and only if for all $k \in N, \pi_{\lambda, \mu}(i, j)=\max _{k \in N} \pi_{\lambda, \mu}(k, j)$ and $\pi_{\lambda, \mu}(i, j)=\min _{k \in N} \pi_{\lambda, \mu}(i, k)$. As in the equal strategic influence case, we investigate the polar case where $\lambda$ tends towards $+\infty$ (while $\mu$ remains fixed) and we have:

$$
\lim _{\lambda \rightarrow+\infty} \pi_{\lambda, \mu}(i, j):=\pi_{\infty, \mu}(i, j)=\frac{\mu\left(1+b_{i}^{j}\right)+n d_{j}\left(1-c_{j}^{i}\right)}{d_{j}\left(1-c_{j}^{i}\right)+\mu}=1+\frac{\mu b_{i}^{j}+(n-1) d_{j}\left(1-c_{j}^{i}\right)}{\mu+d_{j}\left(1-c_{j}^{i}\right)}
$$

So that: 
Proposition 6 A pair of strategies $(i, j)$ is an equilibrium of the game $\mathcal{G}_{\lambda, \mu}$ as $\lambda \rightarrow+\infty$ if and only if for all $k \in N$ :

$$
1+\frac{\mu b_{i}^{j}+(n-1) d_{j}\left(1-c_{j}^{i}\right)}{\mu+d_{j}\left(1-c_{j}^{i}\right)} \leq 1+\frac{\mu b_{i}^{k}+(n-1) d_{k}\left(1-c_{k}^{i}\right)}{\mu+d_{k}\left(1-c_{k}^{i}\right)}
$$

and

$$
1+\frac{\mu b_{i}^{j}+(n-1) d_{j}\left(1-c_{j}^{i}\right)}{\mu+d_{j}\left(1-c_{j}^{i}\right)} \geq 1+\frac{\mu b_{k}^{j}+(n-1) d_{j}\left(1-c_{j}^{k}\right)}{\mu+d_{j}\left(1-c_{j}^{k}\right)}
$$

Again, an interesting particular case is this where $\mu$ tends towards $+\infty$ (while remaining negligible with respect to $\lambda$ ) and one then has:

$$
\pi_{\infty, \mu}(i, j) \rightarrow\left(1+b_{i}^{j}\right)
$$

Hence, $(i, j)$ is an equilibrium if, given $j, b_{k}^{j}$ reaches its maximum in $i$ and, given $i$, $b_{i}^{k}$ reaches its minimum in $j$. In other words, we have a "location game" on the network where the low influence agent must pick up a target that stays clear from the walks leading to its opponent's target while the high influence agent must pick up a target that is the end point of the largest number of walks going through its opponent's target.

For an arbitrary $\mu$, the optimal target takes into account the location and the influenceability of the target of the low influence agent. The lower $\mu$ is, the more important the influenceability of the target is. In a nutshell, if the influence potential is very unequal, the low influence agent shall focus on the margins of the network where highly influenceable agents lay, while the high influence agent shall focus on central nodes of the network, which themselves have a lot of influence.

\subsection{External influenceability and opinion variance}

The measure of influence and influenceability introduced in the preceding section are very demanding computationally. We present here simpler measures that could be used as proxies for actual computations of equilibrium strategies.

As for influenceability, its simplest expression is as follows.

Definition 1 In the uniform model, the external influenceability of an agent $i \in N$ quantifies how much he will take into account the opinion of an external (i.e., strategic) agent and is defined by $1 / d_{i}$.

As for influence, we introduce the following definition.

Definition 2 Let $W$ be an arbitrary weight matrix. The opinion variance of an agent $i \in N$ quantifies to what extent his opinion changes and is defined by

$$
I_{i}=\int_{[0,1]^{n}}\left((W x)_{i}-x_{i}\right)^{2} d x
$$

where $(W x)_{i}$ denotes the ith component of $W x$.

The absolute value could be taken instead in (50), but this would lead to complicated computations. 
Proposition 7 The opinion variance of an agent $i$ is given by

$$
I_{i}=\frac{1}{6}\left(1-w_{i i}\right)^{2}-\frac{1}{6} \sum_{\substack { j \neq i \\
\begin{subarray}{c}{k \neq i \\
k>j{ j \neq i \\
\begin{subarray} { c } { k \neq i \\
k > j } }\end{subarray}} w_{i j} w_{k j}=\frac{1}{12} \sum_{j \neq i} w_{i j}^{2}+\frac{1}{12}\left(1-w_{i i}\right)^{2}
$$

Proof: We have

$$
I_{i}=\int_{[0,1]^{n}}\left(w_{i 1} x_{1}+\cdots+\left(w_{i i}-1\right) x_{i}+\cdots+w_{i n} x_{n}\right)^{2} d x .
$$

Let us prove by weak induction on $n$ that for arbitrary weights $w_{1}, \ldots, w_{n} \in \mathbb{R}$, it holds for any $n \geq 1$ :

$$
\int_{[0,1]^{n}}\left(\sum_{i=1}^{n} w_{i} x_{i}\right)^{2} d x=\frac{1}{3} \sum_{i=1}^{n} w_{i}^{2}+\frac{1}{2} \sum_{i=1}^{n} \sum_{j>i} w_{i} w_{j}
$$

Let us check $P_{1}$.

$$
\int_{0}^{1} w_{1}^{2} x_{1}^{2} d x_{1}=\frac{1}{3} w_{1}^{2}
$$

Then $P_{1}$ is verified, let us suppose $P_{n}$ true and prove $P_{n+1}$. Letting $S(n)=\left(\sum_{i=1}^{n} w_{i} x_{i}\right)^{2}$, we have

$$
\begin{aligned}
\int_{[0,1]^{n+1}} S(n+1) d x_{1} \cdots d x_{n+1}= & \int_{0}^{1}\left(\int_{[0,1]^{n}} S(n+1) d x_{1} \cdots d x_{n}\right) d x_{n+1} \\
= & \int_{0}^{1} \int_{[0,1]^{n}}\left(S(n)+w_{n+1}^{2} x_{n+1}^{2}+2 \sum_{i=1}^{n} w_{i} w_{n+1} x_{i} x_{n+1}\right) d x_{1} \cdots d x_{n} d x_{n+1} \\
= & \int_{0}^{1}\left(\frac{1}{3} \sum_{i=1}^{n} w_{i}^{2}+\frac{1}{2} \sum_{i=1}^{n} \sum_{j=i+1}^{n} w_{i} w_{j}\right) d x_{n+1}+\int_{[0,1]^{n+1}} w_{n+1}^{2} x_{n+1}^{2} d x+ \\
& \int_{0}^{1}\left(\int_{[0,1]^{n}} 2 \sum_{i=1}^{n} w_{i} x_{i} d x_{1} \cdots d x_{n}\right) w_{n+1} x_{n+1} d x_{n+1} \\
= & \frac{1}{3} \sum_{i=1}^{n} w_{i}^{2}+\frac{1}{2} \sum_{i=1}^{n} \sum_{j=i+1}^{n} w_{i} w_{j}+\frac{1}{3} w_{n+1}^{2}+\underbrace{1}_{0}\left(\sum_{i=1}^{n} w_{i}\right) w_{n+1} x_{n+1} d x_{n+1} \\
= & \frac{1}{3} \sum_{i=1}^{n+1} w_{i}^{2}+\frac{1}{2} \sum_{i=1}^{n+1} \sum_{j=i+1}^{n+1} w_{i} w_{j},
\end{aligned}
$$

the desired result.

Applying this to (51), we get, using the fact that $\sum_{j=1}^{n} w_{i j}=1$,

$$
\begin{aligned}
& I_{i}=\frac{1}{3} \sum_{j \neq i} w_{i j}^{2}+\frac{1}{3}\left(w_{i i}-1\right)^{2}+\frac{1}{2} \sum_{\substack { j \neq i \\
\begin{subarray}{c}{k \neq i \\
k>j{ j \neq i \\
\begin{subarray} { c } { k \neq i \\
k > j } }\end{subarray}} w_{i j} w_{i k}+\underbrace{\frac{1}{2} \sum_{j \neq i}\left(w_{i i}-1\right) w_{i j}}_{-\frac{1}{2}\left(1-w_{i i}\right)^{2}} \\
& =\frac{1}{3} \sum_{j \neq i} w_{i j}^{2}-\frac{1}{6}\left(w_{i i}-1\right)^{2}+\frac{1}{2} \sum_{\substack{j \neq i \\
j \neq i}} \sum_{\substack{k \neq i \\
k>j}} w_{i j} w_{i k} .
\end{aligned}
$$


Now,

$$
\left(1-w_{i i}\right)^{2}=\left(\sum_{j \neq i} w_{i j}\right)^{2}=\sum_{j \neq i} w_{i j}^{2}+2 \sum_{\substack { j \neq i \\
\begin{subarray}{c}{k \neq i \\
k>j{ j \neq i \\
\begin{subarray} { c } { k \neq i \\
k > j } }\end{subarray}} w_{i j} w_{i k}
$$

from which it follows that

$$
I_{i}=\frac{1}{6}\left(1-w_{i i}\right)^{2}-\frac{1}{6} \sum_{\substack{j \neq i \\ j \neq i \\ k>j}} \sum_{\substack{k \neq i \\ k>j}} w_{i k}
$$

or

$$
I_{i}=\frac{1}{12} \sum_{j \neq i} w_{i j}^{2}+\frac{1}{12}\left(1-w_{i i}\right)^{2}
$$

Proposition 8 The opinion variance $I_{i}$ takes value in $\left[0, \frac{1}{6}\right]$. The minimum is achieved when $w_{i i}=1$ and the maximum is achieved if and only if $w_{i j}=1$ for some $j \neq i$.

Proof: The result for the lower bound is obvious. As for the upper bound, taking (52), the upper bound is attained iff the first term is maximized and the second is minimized. Taking $w_{i i}=0$ maximizes the first term. Now the second term is 0 iff all but one weight among $w_{i j}, i \neq j$, is nonzero.

Example 5 In Example 2, the external influenceabilities of the non-strategic agents are equal to 1 for agent 1 , and $\frac{1}{3}$ for agents 2 and 3 . Their opinion variances are equal to $I_{1}=0, I_{2}=I_{3}=1 / 18$.

\subsection{The case of a single strategy agent}

Before concluding, let us point out that in the case of a single strategic agent, the optimal targeting problem is trivial in our framework. Suppose indeed that there exists only one strategic agent $a_{1}$ who has the extreme opinion equal to 1 . As before we can use the results recapitulated in Section 3 on non-negative matrices and applied in Section 4 to the model with two strategic agents. If by $P$ we denote the $(n+1) \times(n+1)$ matrix of weights of the agents in $N$ and $a_{1}$, then there is one essential class of $P$, i.e., $\left\{a_{1}\right\}$, and all previous classes of $W$ become inessential. The structure of $P$ is therefore

$$
P=\left[\begin{array}{l|l}
1 & \mathbf{0} \\
\hline R & Q
\end{array}\right]
$$

As before, if $s_{1}=i$, then the weight $w_{i j}=\frac{a_{i j}}{d_{i}}$ becomes $\frac{1}{d_{i}+\lambda}$, and the weight on $a_{1}$ is equal to $w_{s_{1} a_{1}}=\frac{\lambda}{d_{s_{1}}+\lambda}$. We have

$$
P=\left[\begin{array}{c|c}
1 & \mathbf{0} \\
\hline \Delta\left(s_{1}\right) E\left(s_{1}\right) & \Delta\left(s_{1}\right) A
\end{array}\right]
$$


with $E\left(s_{1}\right)=\lambda e_{s_{1}}$. If the opinion vector $x$ is written as $x=\left[\begin{array}{ll}x_{a_{1}} & x_{N}\end{array}\right]^{\prime}$, then the opinions of non-strategic agents are updated as follows:

$$
x_{N}(t+1)=R+Q x_{N}(t)
$$

and for the uniform model:

$$
x_{N}(t+1)=\Delta\left(s_{1}\right) A x_{N}(t)+\frac{\lambda}{d_{s_{1}}+\lambda} e_{s_{1}}
$$

As before, $\bar{x}_{a_{1}}=1$, convergence of $x_{N}$ always occurs and $\bar{x}_{N}=(I-Q)^{-1} R$. All agents in $N$ reach consensus equal to 1 . Consequently, the payoff of the strategic agent $a_{1}$ is equal to $\pi_{\lambda}\left(s_{1}\right)=n$, independently of which non-strategic agent $i$ is targeted by $a_{1}$.

\section{Discussion and concluding remarks}

In the paper we investigate a model of opinion (belief) formation with two kinds of agents and some strategic aspects of influence. Our point of departure is the model of DeGroot (1974). Apart from the non-strategic agents that interact as in the DeGroot model, in our framework there exist additionally two strategic agents with opposite opinions who target the non-strategic agents in order to influence their opinions. We model the issue as a zero-sum game played by the two strategic agents. Our results concern convergence and the characterization of equilibria in the proposed model.

They emphasize two features that are crucial to choose an optimal target in a strategic setting. On the one hand, the influenceability of the agent measured by (the inverse of) its number of outgoing arcs, i.e., the number of agents that he listens to. On the other hand, the influence of the agent for which we introduce a new measure, that can be called intermediacy and that counts the number of walks that go through a given agent. In the case where the two strategic agents have the same impact, we show that the relative importance of intermediacy increases with the actual impact the strategic agents exert and decreases with the size of the network. In the case where the relative impact of one of the strategic agents is much greater than this of the other, the high impact agent should focus on high influence/intermediacy targets and the low impact one on high influenceability targets.

The framework proposed in the paper (both with two as well as with only one strategic agent) can model naturally many real-life situations of influence, like leadership of some individuals which influence other members of a group (community, society) while remaining not 'influenceable' themselves. The non-strategic agents can form, for instance, the panel of experts/decision makers who must make a decision after discussion. The strategic agents try to influence the final result at their advantage by targeting one of the experts/decision makers. Similarly, one might consider an influence by media sources, targeting consumers in a process of products promotion, influencing voters in political campaigns, etc. As targeting some individuals is being used rather widely in practice, it could be interesting to test our model against empirical data from social networks and with the support of experiments. Which strategies do 'strategic subjects' play and which players do they target in order to influence their opinions? Do their choices of 'non-strategic subjects' correspond to our results? 
From a more theoretical point of view, our approach is based on the implicit assumption that the formation of an influence link is a very costly process so that the strategic agents can only form one link and cannot change it over time. Relaxing both assumptions would certainly yield new interesting insights. 


\section{Bibliography}

D. Acemoglu and A. Ozdaglar. Opinion dynamics and learning in social networks. Dynamic Games and Applications, 1:3-49, 2011.

D. Acemoglu, A. Ozdaglar, and A. ParandehGheibi. Spread of (mis)information in social networks. Games and Economic Behavior, 70:194-227, 2010.

D. Acemoglu, M. Dahleh, I. Lobel, and A. Ozdaglar. Bayesian learning in social networks. Review of Economic Studies, 78:1201-1236, 2011.

D. Acemoglu, G. Como, F. Fagnani, and A. Ozdaglar. Opinion fluctuations and disagreement in social networks. Mathematics of Operations Research, 38(1):1-27, 2013.

V. Bala and S. Goyal. Learning from neighbours. The Review of Economic Studies, 65(3): 595-621, 1998.

C. Ballester, A. Calvo-Armengol, and Y. Zenou. Who's who in networks. Wanted: The key player. Econometrica, 74(5):1403-1417, 2006.

A. Banerjee, A. Chandrasekhar, E. Duflo, and M. Jackson. Diffusion of microfinance. Science, 341, DOI: 10.1126/science.1236498, 2013.

A. Banerjee, A. Chandrasekhar, E. Duflo, and M. Jackson. Gossip: Identifying central individuals in a social network. MIT Department of Economics Working Paper No. $14-15,2014$.

A. V. Banerjee. A simple model of herd behavior. Quarterly Journal of Economics, 107(3):797-817, 1992.

A. V. Banerjee and D. Fudenberg. Word-of-mouth learning. Games and Economic Behavior, 46:1-22, 2004.

S. Bikhchandani, D. Hirshleifer, and I. Welch. A theory of fads, fashion, custom, and cultural change as informational cascades. Journal of Political Economy, 100:992-1026, 1992.

P. B. Bonacich. Power and centrality: a family of measures. American Journal of Sociology, 92:1170-1182, 1987.

B. Büchel, T. Hellmann, and M. Pichler. The dynamics of continuous cultural traits in social networks. Journal of Economic Theory, 154:274-309, 2014.

B. Büchel, T. Hellmann, and S. Klößner. Opinion dynamics and wisdom under conformity. Journal of Economic Dynamics and Control, Forthcoming.

P. Clifford and A. Sudbury. A model for spatial conflict. Biometrika, 60(3):581-588, 1973.

G. Deffuant, D. Neau, F. Amblard, and G. Weisbuch. Mixing beliefs among interacting agents. Advances in Complex Systems, 3(01n04):87-98, 2000.

M. H. DeGroot. Reaching a consensus. Journal of the American Statistical Association, 69:118-121, 1974.

P. DeMarzo, D. Vayanos, and J. Zwiebel. Persuasion bias, social influence, and unidimensional opinions. Quarterly Journal of Economics, 118:909-968, 2003.

P. Domingos and M. Richardson. Mining the network value of customers. Seventh International Conference on Knowledge Discovery and Data Mining, pages 57-66, 2001.

P. Duersch, J. Oechssler, and B. C. Schipper. Pure strategy equilibria in symmetric two-player zero-sum games. International Journal of Game Theory, 41(3):553-564, 2012. 
R. Durrett. Lecture Notes on Particle Systems and Percolation. Wadsworth Publishing, 1988.

G. Ellison and D. Fudenberg. Rules of thumb for social learning. Journal of Political Economy, 101(4):612-643, 1993.

M. Förster, M. Grabisch, and A. Rusinowska. Anonymous social influence. Games and Economic Behavior, 82:621-635, 2013.

N. E. Friedkin and E. C. Johnsen. Social influence and opinions. Journal of Mathematical Sociology, 15:193-206, 1990.

N. E. Friedkin and E. C. Johnsen. Social positions in influence networks. Social Networks, 19:209-222, 1997.

A. Galeotti and S. Goyal. Influencing the influencers: a theory of strategic diffusion. The RAND Journal of Economics, 40(3):509-532, 2009.

A. Galeotti, C. Ghiglino, and F. Squintani. Strategic information transmission networks. Journal of Economic Theory, 148(5):1751-1769, 2013.

R. J. Glauber. Time-dependent statistics of the Ising model. Journal of Mathematical Physics, 4:294-307, 1963.

B. Golub and M. O. Jackson. Naïve learning in social networks and the wisdom of crowds. American Economic Journal: Microeconomics, 2(1):112-149, 2010.

M. Grabisch and A. Rusinowska. A model of influence based on aggregation functions. Mathematical Social Sciences, 66:316-330, 2013.

M. Granovetter. Threshold models of collective behavior. American Journal of Sociology, 83:1420-1443, 1978.

J. Hagenbach and F. Koessler. Strategic communication networks. Review of Economic Studies, 77:1072-1099, 2010.

R. Hegselmann and U. Krause. Opinion dynamics and bounded confidence, models, analysis and simulation. Journal of Artificial Societies and Social Simulation, 5(3): $1-33,2002$.

M. O. Jackson. Social and Economic Networks. Princeton University Press, 2008.

D. Kempe, J. Kleinberg, and E. Tardos. Maximizing the spread of influence through a social network. Proceedings of 9th International Conference on Knowledge Discovery and Data Mining, pages 137-146, 2003.

D. Kempe, J. Kleinberg, and E. Tardos. Influential nodes in a diffusion model for social networks. Proceedings of 32nd International Colloquium on Automata, Languages and Programming, pages 1127-1138, 2005.

U. Krause. A discrete nonlinear and nonautonomous model of consensus formation. In S. Elaydi, G. Ladas, J. Popenda, and J. Rakowski, editors, Communications in Difference Equations. Amsterdam: Gordon and Breach, 2000.

T. M. Liggett. Interacting Particle Systems. Springer, 1985.

J. Lorenz. A stabilization theorem for dynamics of continuous opinions. Physica A, 355: 217-223, 2005.

J. Lorenz. Convergence of products of stochastic matrices with positive diagonals and the opinion dynamics background. In C. Commault and N. Marchand, editors, Positive Systems, volume 341 of Lecture Notes in Control and Information Sciences, pages 209216. Springer Berlin / Heidelberg, 2006.

M. Richardson and P. Domingos. Mining knowledge-sharing sites for viral marketing. Eighth International Conference on Knowledge Discovery and Data Mining, page 6170, 2002 . 
T. Schelling. Micromotives and Macrobehavior. Norton, 1978.

E. Seneta. Non-negative Matrices and Markov Chains. Springer Series in Statistics, Springer, 2006. 